Doi: $10.4274 /$ jus.2019.06.007

\title{
Re: Cannabinoids as an Anticancer Agent for Prostate Cancer
}

Domenica Roberto1, Laurence H Klotz², Vasundara Venkateswaran1,2

${ }^{1}$ Toronto University, Institute of Medical Science, Toronto, Canada

2Sunnybrook Health Sciences Centre, Department of Surgery, Toronto,

J Urol Res 2017:4:1090.

\section{EDITORIAL COMMENT}

Cannabis has been used for medical purposes for more than 5000 years. Over the past decade, the endocannabinoid system has emerged as a novel target for the treatment, prevention of cancer and various diseases. Cannabinoids consist of the active components of the plant Cannabis sativa. They can be classified into three groups based on the source of their production: phytocannabinoids, endogenous cannabinoids, and synthetic cannabinoids. Their effects are mainly mediated via the activation of two G-protein coupled cannabinoid (CB) receptors i.e. CB1 and CB2. Recently, the therapeutic efficacy of cannabinoids have been reported to be due to palliative (antiemetic and analgesic) and antitumoral (antiproliferative and anti-metastatic) effects in many cancers. Overexpression of cannabinoid receptors in malignant prostate tissue can be an alternative option for the treatment of prostate cancer. In this research, the authors suggested positive results associated with potential apoptotic and anti-metastatic effects due to endoplasmic reticulum stress, oxidative stress and Rho GTPase signaling pathways. There is need for additional studies about carcinogenesis of the prostate cancer especially in vivo using xenograft and/or transgenic models to investigate the role of cannabinoids in the treatment. Cannabinoid treatment has safety toxicological prolife and it is a logical candidate for use in combination therapy with chemotherapy. This review provides support for the treatment of prostate cancer with Cannabinoids as an anticancer agent. In the future, treatment and prevention of prostate cancer with Cannabinoids due to targeting the endocannabinoid system may be preferable. Further research are warranted.

Fehmi NARTER, MD, PhD

๑Copyright 2019 by the Association of Urological Surgery / Journal of Urological Surgery published by Galenos Publishing House. 